\title{
Alcohol septal ablation in hypertrophic cardiomyopathy leading to permanent complete heart block; a case report
}

\author{
Mendis SAES, Priyadarshan P, SeneviratneNHG, Ambiga K, Herath RRGCSB \\ Institute of Cardiology, National Hospital of Sri Lanka, Colombo, Sri Lanka.
}

Correspondence: Dr. Sepalika Mendis

e-mail: sepalikamendis@yahoo.com

\section{Introduction}

Hypertrophic cardiomyopathy (HCM) is a disease state characterised by unexplained left ventricular hypertrophy in the absence of another cardiac or systemic disease which can explain it (1). HCM is inherited as an autosomal dominant trait and has diverse clinical manifestations ranging from asymptomatic patients with normal life span to sudden cardiac death (2). A considerable proportion of patients with HCM have left ventricular outflow tract obstruction (LVOT) at rest or on provocation. Medical management of HCM involves beta blockers and calcium channel blockers. Patients with LVOT obstruction, refractory to medical management are considered for septal reduction therapy with alcohol septal ablation (ASA) or myomectomy. Complete heart block is a known complication of ASA which is usually transient. We report a HCM case with LVOT obstruction needing permanent pacing following ASA.

\section{Case report}

In 2005, a 51-year-old male presented with shortness of breath on moderate exertion and systolic murmur was found on clinical examination. His ECG revealed bizarre ST and $\mathrm{T}$ wave changes. Transthoracic echocardiogram (TTE) confirmed the diagnosis of HCM with interventricular septum diameter of $20 \mathrm{~mm}$, LVOT obstruction of $85 \mathrm{mmHg}$ and systolic anterior motion of mitral valve. $\mathrm{He}$ was started on beta blockers following which the patient became asymptomatic. Meanwhile, the family screening with echocardiography revealed negative results.
In January 2013, patient presented with worsening dyspnoea accompanying chest pain on moderate exertion. He underwent coronary angiogram which showed a prominent first septal branch in left anterior descending artery (LAD). There was no evidence of syncopy and his left ventricular (LV) ejection fraction was satisfactory during the course of illness. Since he continued to be symptomatic despite maximum medical therapy it was decided to proceed with ASA.

Pre procedure simultaneous pressure recordings at LVOT and LV apex revealed a difference of $72 \mathrm{mmHg}$. We proceeded with the coronary angiogram to identify the first septal perforator of LAD which was subsequently engaged with $2 \mathrm{~mm}$ over the wire balloon (OTW). With the inflation of OTW balloon the pressure gradient across LVOT and LV reduced dramatically to $20 \mathrm{mmHg}$. Area supplied by the first septal branch and absence of backflow to LAD were confirmed with contrast injection. A temporary pace maker (TPM) lead was placed in the right ventricular apex anticipating atrioventricular blocks. $1 \mathrm{ml}$ of $100 \%$ alcohol was slowly injected with $0.5 \mathrm{ml}$ at a time and kept for 15 minutes. There was a dramatic reduction in pressure gradient across the LVOT $(4 \mathrm{mmHg})$ and the patent went into complete heart block and became dependent on TPM.

During the seven day stay at coronary care unit, the patient continued to have intermittent complete heart block and was TPM dependent. After consulting cardiac electrophysiology team, it was decided to insert a permanent dual chamber pacemaker. However following the insertion of permanent pacemaker he became asymptomatic and gradually 
resumed his usual life style. TTE done 1month later revealed only $30 \mathrm{mmHg}$ pressure gradient across the LVOT which is expected to reduce in next few months.

\section{Discussion}

ASA is accepted as a reliable modality of treatment for symptom relief in patients with HCM since $1994(1,3)$. The indications for ASA are refractory symptoms despite medical treatment, resting or provocable pressure gradient across LVOT more than $50 \mathrm{mmHg}$ and inter-ventricular diameter more than $18 \mathrm{~mm}$. As our patient fulfilled these criteria we considered him for ASA $(1,3)$.

Tributaries of the first septal branch of the LAD have significant individual variations. They supply a large area of myocardium including right ventricular septum and the LV apex. Therefore, the exact localisation of the septal branch supplying basal septum is paramount in ASA. Anatomical variations of $\mathrm{LAD}$ can result in post procedural atrioventricular conduction defects but they usually recover spontaneously within 24 - 48 hours while $10-20 \%$ will persist and require permanent pacing (1). The myocardial contrast echocardiography (MCE) during procedure gives an opportunity to select the target septal branch in each individual, minimizing above complication $(3,4)$. Thus the reduction of the incidences of post procedure pacemaker implantation is a proven advantage of MCE guided ASA which is yet to establish in our country (4).
Alternatively the dual chamber pacing is considered as an effective mode of treatment for symptom relief in HCM (1). The right ventricular apical pacing with the maintenance of atrioventricular synchrony has been reported to decrease the LVOT pressure gradient. Therefore, our patient was benefitted in both aspects with permanent pacing.

\section{References}

1. ACCF/AHA guideline for the diagnosis and treatment of Hypertrophic cardiomyopathy 2011. A report of the American College of Cardiology foundation / American Heart Association Task force on practice guideline (2011). Journal of the American College of Cardiology, 2011; 58(25): e213-e260.

2. Agarwal SS, Choksi C, Sheikh I. Sudden death - A case Hypertrophic Cardiomyopathy: JIAFM, 2004; 26(4) ISSN 0971-0973.

3. Marsy EJ, Breall JA. Alcohol septal Ablation for Hypertrophic Cardiomyopathy: Curr Cardial Rev, 2008; 4(3): 193-7.

4. Faber L, Seggewiss H, Gleichmann U. Percutaneous Transluminal Septal Myocardial Ablation in Hypertrophic cardiomyopathy. Results with respect to intraprocedural myocardial contrast echocardiography: Circulation, January 1999 2415-2421.

Available in http://www.researchgate.net/ publication/ 13453283 